\title{
Tumor Necrosis Factor Receptor Associated Factor 3 Modulates Cartilage Degradation through Suppression of Interleukin 17 Signaling
}

\author{
Guoli Hu, ${ }^{* \dagger \ddagger}$ Ning Zhang, ${ }^{\star \dagger \S}$ Jiao Li, ${ }^{* \dagger}$ Jing Wang, Wen Wu, Jing Li, ${ }^{* \dagger}$ Wenxue Tong, ${ }^{\star \dagger}$ Xiaoying Zhao, ${ }^{* \dagger}$ Liming Dai, ${ }^{*}$ and \\ Xiaoling Zhang ${ }^{* \dagger}$
}

\begin{abstract}
From the Department of Orthopedic Surgery, * Xin Hua Hospital, Shanghai Jiao Tong University School of Medicine, Shanghai, China; The Key Laboratory of Stem Cell Biology, ${ }^{\dagger}$ Shanghai Institute for Biological Sciences, Chinese Academy of Sciences, Shanghai, China; the Department of Orthopedic Surgery, ${ }^{\ddagger}$ Duke University School of Medicine, Durham, North Carolina; the Department of Orthopedic Surgery, ${ }^{\S}$ Stanford University School of Medicine, Redwood City, California; and the Shanghai Key Laboratory of Orthopedic Implant, ${ }^{\circledR}$ Department of Orthopedics, Shanghai Ninth People’s Hospital, Shanghai Jiao Tong University School of Medicine, Shanghai, China
\end{abstract}

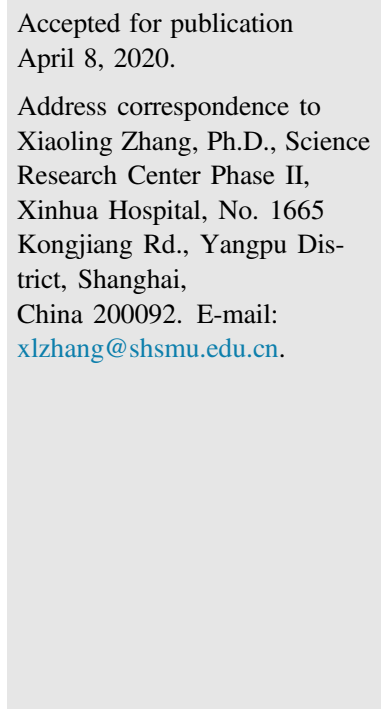

Accepted for publication

April 8, 2020.

Address correspondence to Xiaoling Zhang, Ph.D., Science Research Center Phase II, Yangpu DisChina 200092. E-mail: xlzhang@shsmu.edu.cn.

\begin{abstract}
Interleukin 17A (IL-17A) is critical in the pathogenesis of autoimmune diseases through driving inflammatory cascades. However, the role of IL-17 in osteoarthritis (OA) is not well understood. Tumor necrosis factor-receptor-associated factor 3 (TRAF3) is a receptor proximal negative regulator of IL-17 signaling. It remains unclear whether TRAF3 exerts regulatory effects on cartilage degradation and contributes to the pathogenesis of OA. In this study, we found that TRAF3 notably suppressed IL-17 -induced NF- $\mathrm{KB}$ and mitogen-activated protein kinase activation and, subsequently, the production of matrix-degrading enzymes. TRAF3 depletion enhanced IL-17 signaling, along with increased matrixdegrading enzyme production. In vivo, cartilage destruction caused by surgery-induced $\mathrm{OA}$ was alleviated markedly both in 1l17a-deficient mice and in TRAF3 transgenic mice. In contrast, silencing TRAF3 through adenoviruses worsened cartilage degradation in experimental OA. Moreover, the destructive effect of IL-17 on cartilage was abolished in TRAF3 transgenic mice in an IL-17 intraarticular injection animal model. Similarly, genetic deletion of IL-17 blocked TRAF3 knockdown-mediated promotion of cartilage destruction, suggesting that the protective effect of TRAF3 on cartilage is mediated by its suppression of IL-17 signaling. Collectively, our results suggest that TRAF3 negatively regulates IL-17-mediated cartilage degradation and pathogenesis of $0 \mathrm{~A}$, and may serve as a potential new therapy target for OA. (Am J Pathol 2020, 190: 1701-1712; https:// doi.org/10.1016/j.ajpath.2020.04.016)
\end{abstract}

Osteoarthritis (OA) is the most common joint disease characterized by cartilage destruction, subchondral bone sclerosis, and synovial inflammation. ${ }^{1}$ These abnormities have been attributed mainly to an imbalance between the anabolism and catabolism of the articular cartilage, especially an increase in catabolism. ${ }^{2}$ Proinflammatory cytokines, important mediators of OA, such as interleukin (IL)-1 $\beta$, tumor necrosis factor (TNF)- $\alpha$, and IL-6, are widely investigated in articular cartilage. $^{3-5}$ IL-17 is a crucial proinflammatory factor in the pathogenesis of various autoimmune diseases, such as

\footnotetext{
Supported by National Natural Science Foundation of China grants 81830078 (X.Z.), 81772347 (X.Z.), 81572123 (X.Z.), Science and Technology Commission of Shanghai Municipality grant 19XD1434100 (X.Z.), Shanghai Municipal Education Commission-Gao feng Clinical Medicine Grant Support 20161314 (X.Z.), Shanghai Jiao Tong University International Collaboration Project, and the Shanghai Jiao Tong University-The Chinese University of Hong Kong Joint Research Collaboration Fund (X.Z.).

G.H. and N.Z. contributed equally to this work.

Disclosures: The authors have submitted a patent on TRAF3 modulates cartilage degradation (patent application number 202010350036.8).
} 
experimental autoimmune encephalomyelitis, ${ }^{6}$ collageninduced arthritis, ${ }^{7}$ and inflammatory bowel disease. ${ }^{8}$ IL-17 is a major inflammation-driving cytokine, exerting its functions through inducing and sustaining the production of inflammatory cytokines, chemokines, and matrix metalloproteinases. ${ }^{9}$ IL-17 also can act synergistically with IL-1 $\beta$ or TNF- $\alpha$ for further induction of proinflammatory genes. ${ }^{10,11}$ In rheumatoid arthritis, synovial fibroblast IL-17 has been proven important because it induces the production of inflammatory factors IL- 6 and IL- 8 via the NF- $\kappa$ B and phosphatidylinositol 3-kinase/Akt pathways. ${ }^{12}$ During OA, IL-17 is an upstream factor in the regulation of IL-1 $\beta$ and TNF- $\alpha .{ }^{4,13}$ In articular cartilage and chondrocytes, IL-17 up-regulates the mRNA expression of several cartilage catabolic factors such as IL-6, nitric oxide, and matrix metalloproteinases (MMPs) through NF- $\kappa \mathrm{B}$ and mitogenactivated protein kinase (MAPK) pathways. ${ }^{13-15}$ IL-17 also is more active in chondrocytes than in fibroblasts during OA pathophysiology. ${ }^{16}$ Interestingly, significant increases in circulating $\mathrm{T}$ helper 17 cells were observed in patients after anti-TNF- $\alpha$ therapy. Thus, the combined blockade of TNF- $\alpha$ and IL-17 in experimental arthritis was more effective than monotherapy. ${ }^{17}$ Therefore, blocking the IL-17 pathway is a promising method for preventing cartilage from degradation in arthritis.

The TNF-associated factors (TRAFs) are a family of intracellular adaptor proteins that mediate downstream signaling of various cell surface receptors and thus control the early stages of cellular defense mechanisms against invading bacterial and viral pathogens. Most members of this family, including TRAF2, TRAF5, and TRAF6, have been studied intensively, however, investigations on TRAF3 remain scarce. ${ }^{18}$ The upregulation of TRAF3 expression in osteoclast precursors can mitigate bone destruction and inflammation-induced bone loss in common bone diseases. ${ }^{19}$ Moreover, TRAF3 is a critical factor in controlling the induction of type I interferon ${ }^{20}$ and the expression of the anti-inflammatory cytokine IL- $10 .^{21}$ These findings suggest that TRAF3 plays a complicated role in restoring immunologic homeostasis. Recent reports have indicated that TRAF3 could play a negative role in IL-17-induced NF- $\kappa B$ and MAPK signaling pathways and that knockdown of TRAF3 greatly enhanced IL-17 signaling in human primary synoviocytes. ${ }^{22}$ As previously mentioned, IL-17 has a greater influence on chondrocytes than synoviocytes during OA process. ${ }^{16}$ The effect of TRAF3 on chondrocytes and protecting cartilage from degradation has yet to be investigated further.

In this study, we showed that overexpression of TRAF3 significantly blocked IL-17-induced NF- $\kappa \mathrm{B}$ and MAPK pathways in chondrocytes and consequently reduced the production of IL-6 and MMPs. Conversely, knocking down TRAF3 resulted in increased MMP-13 production induced by IL-17. In vivo, loss of IL-17 markedly alleviated cartilage destruction caused by surgery-induced OA and age-related spontaneous OA. TRAF3 transgenic mice showed less cartilage destruction in different arthritic animal models compared with littermate control. Furthermore, silencing TRAF3 by using adenoviruses markedly worsened cartilage degradation in experimental OA, which partially was rescued by IL-17 deficiency. Collectively, TRAF3 is likely to be a potential therapeutic target for ameliorating arthritis cartilage degradation.

\section{Materials and Methods}

\section{Animals}

TRAF3 transgenic mice and $I l 17 a-$ deficient $\left(I l 17 a^{-/-}\right)$mice were a kind gift from Professor Youcun Qian from the Shanghai Institutes for Biological Sciences (Chinese Academy of Sciences, Shanghai, China). The generation of TRAF3 transgenic mice was described in a previous study. ${ }^{22}$ The TRAF3 transgenic mice (T3TG) and $1117 a^{-1-}$ mice and littermate [wild-type (WT)] controls at 8 to 12 weeks of age were used for experiments. C57BL/6 mice and Sprague Dawley rats were purchased from the Shanghai Laboratory Animal Center (Chinese Academy of Sciences). All of the animals were maintained under pathogen-free conditions. All of the animal experiments were performed in compliance with the Guide for the Care and Use of Laboratory Animals and were approved by the Institutional Biomedical Research Ethics Committee of the Shanghai Institutes for Biological Sciences (Chinese Academy of Sciences).

\section{IL-17 Intra-Articular Injection Model}

Mice were anesthetized with $250 \mathrm{mg} / \mathrm{kg}$ intraperitoneal tribromoethanol (Sigma, St. Louis, MO). A total volume of $5 \mu \mathrm{L}$ containing $10 \mathrm{ng} / \mathrm{mL}$ recombinant IL-17 (R\&D, McKinley, MN) in phosphate-buffered saline (PBS) was injected through the infrapatellar ligament into the left knee joint space using a $30 \mathrm{G}$ needle (65460-05; Hamilton Co., Bonaduz, Switzerland) as previously described, ${ }^{23}$ and the patellar ligament was made visible through a small skin incision. The right knee was injected with $5 \mu \mathrm{L}$ PBS as a control group. An intra-articular injection of IL-17 $(10 \mathrm{ng} / \mathrm{mL}$ in PBS) and PBS into the T3TG mice and littermate controls three consecutive times every 24 hours was performed. Three days after the last injection, T3TG and WT mice were euthanized through $\mathrm{CO}_{2}$ administration ( $n=10$ per group). The samples of the knee joints were collected and fixed in $4 \%$ paraformaldehyde.

\section{Destabilization of the Medial Meniscus Model and Osteoarthritis Research Society International Scoring}

Mice destabilization of the medial meniscus (DMM) was induced by transection of the anterior attachment of the medial meniscus to the tibia plateau. ${ }^{24,25}$ Knees then were prepared for aseptic surgery. A longitudinal incision medial to the patellar ligament was made, the joint capsule was opened, and the meniscotibial ligament, which anchors the medial meniscus to the tibial plateau, was identified. No 
further manipulation was performed on a subset of animals considered as the sham group. In the experimental group, the medial meniscotibial ligament was transected, resulting in DMM. In both sham and DMM animals, the joint capsule and subcutaneous layer were sutured and closed separately. Six weeks after DMM, T3TG and WT mice were euthanized through $\mathrm{CO}_{2}$ administration. For adenovirus injection, control viruses (Ad-C) or adenoviruses containing TRAF3 short hairpin RNA plasmid (Ad-sh-Traf3) viruses were injected intra-articularly once a week 1 week after DMM surgery for 5 weeks. The knee joints were collected and fixed in $10 \%$ buffered formalin overnight at $4^{\circ} \mathrm{C}$, followed by 2 -week decalcification in 14\% EDTA. Joints then were embedded in paraffin and sectioned at 5- $\mu \mathrm{m}$ thickness. Histologic slides were prepared and sections were stained using safranin-O (S2255; Sigma) and fast green (F7252; Sigma), and then graded for OA severity by four independent graders using the Osteoarthritis Research Society International scoring system. ${ }^{26,27}$ Three sections (medial, central, and lateral) from each joint were stained and evaluated. The three sections were graded separately and averaged for each joint.

\section{Rat OA Animal Model Human Cartilage Sample}

Rat OA was induced by DMM surgery as described in the previous paragraph. Briefly, the animals were anesthetized, and surgery was performed to transect the medial collateral ligament. The medial meniscus was cut through the full thickness to induce the joint destabilization of the right knee. Sham animals underwent the same surgical procedure without any ligament transection or meniscal tear as the control group. The rats that underwent surgery were euthanized at 1,2 , or 3 months after surgery ( $n=7$ to 10 per group), the samples of the knee joints were collected and fixed in $4 \%$ paraformaldehyde. Human OA cartilage was sampled from patients who underwent a total joint replacement because of primary OA of the knee $(N=3)$. Undamaged areas in the same patient were sampled as the normal cartilage. Ethical approval was obtained from the Medical Ethics Committee of the Shanghai Ninth People's Hospital (Shanghai Jiao Tong University School of Medicine, Shanghai, China), and informed consent was obtained from all participants.

\section{Histology and Immunohistochemistry}

Knee joints isolated from the experimental animals were fixed overnight with $4 \%$ paraformaldehyde decalcified by $12.5 \%$ EDTA in PBS (pH $=7.0)$ and then embedded in paraffin. Tissue sections $(5 \mu \mathrm{m})$ were deparaffinized in xylene, serially rehydrated in ethanol, and washed with PBS. Sections were stained with safranin $\mathrm{O} /$ fast green to identify proteoglycan loss. Safranin O staining was quantified according to density using ImageJ software version 1.52t (NIH, Bethesda, MD; https:// imagej.nih.gov/ij). For immunohistochemistry, sections in 10 $\mathrm{mmol} / \mathrm{L}$ sodium citrate buffer $(\mathrm{pH}$ 6.0) were heated in a microwave oven at $95^{\circ} \mathrm{C}$ for 10 minutes. Slides were cooled for 30 minutes at room temperature after antigen unmasking. Endogenous peroxidase activity was blocked with $3 \%$ hydrogen peroxide and was rinsed several times with PBS. After blocking nonspecific protein binding with 5\% bovine serum albumin in PBS for 30 minutes at room temperature, sections were incubated overnight at $4^{\circ} \mathrm{C}$ with primary antibodies against TRAF3 (ab217033; Abcam, Cambridge, UK) or MMP-13 (ab219620; Abcam). The slides were rinsed with PBS and then incubated with secondary antibody (Cell Signaling Technology, Danvers, MA) in accordance with the manufacturer's instructions. The sections then were counterstained with Mayer's hematoxylin (Sigma). After being washed, the slides were stained with 3,3'-diaminobenzidine tetrahydrochloride (EnVision detection kit, peroxidase/diaminobenzidine, rabbit/mouse; Dako Cytomation, Santa Clara, CA). Staining with normal IgG and staining without primary antibodies also was performed as a negative control. The immunohistochemistry results were quantified through calculating the percentage of positive cells using ImageJ software.

\section{Cell Culture}

\section{Rat Chondrocytes Isolation and Culture}

Primary rat chondrocytes were isolated from the rats' knee cartilage, after digestion by type II collagenase for 5 hours at $37^{\circ} \mathrm{C}$, filtered using a 70- $\mu \mathrm{m}$ filter (BD Corning, Franklin Lakes, NJ), and then cultured in a mixture of Dulbecco's modified Eagle's medium and Ham's F12 medium (Thermo Fisher Scientific, Waltham, MA) with $10 \%$ fetal bovine serum (Thermo Fisher Scientific), $100 \mathrm{U} / \mathrm{mL}$ penicillin G, $100 \mu \mathrm{g} / \mathrm{mL}$ streptomycin, and $2.5 \mu \mathrm{g} / \mathrm{mL}$ amphotericin B under a humidified atmosphere of $5 \% \mathrm{CO}_{2}$ in air at $37^{\circ} \mathrm{C}$.

\section{Chondrogenic Cell Line ATDC5}

The murine chondrogenic ATDC5 cell line was obtained from RIKEN Cell Bank (Tsukuba, Japan). Cultures of undifferentiated ATDC5 cells were maintained in a mixture of Dulbecco's modified Eagle's medium and Ham's F12 medium and supplemented with $10 \%$ fetal bovine serum, $100 \mathrm{U} / \mathrm{mL}$ penicillin $\mathrm{G}, 100 \mu \mathrm{g} / \mathrm{mL}$ streptomycin, and $2.5 / \mathrm{mL}$ amphotericin B.

\section{Chondrosarcoma Cell Line SW1353}

The human chondrosarcoma cell line SW1353 cells were obtained from the Type Culture Collection of the Chinese Academy of Sciences. The cells were cultured similarly in a mixture of Dulbecco's modified Eagle's medium and Ham's F12 medium.

\section{Transient Transfections Gene Expression}

Flag-tagged TRAF3 was cloned into a retrovirus-mediated pMSCV-IRES-GFP vector (kindly provided by Youcun Qian's laboratory, Institute of Health Science, Shanghai Institute for Biological Sciences, Chinese Academy of Sciences, Shanghai, China) and a pcDNA 3.0 plasmid. ATDC5 
A

Primary chondrocytes
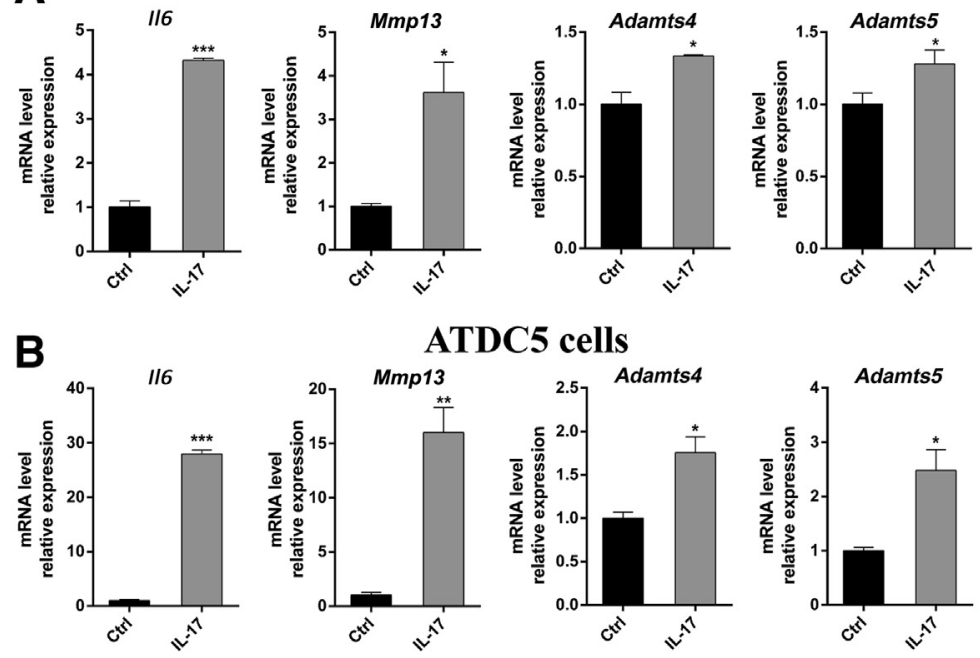

ATDC5 cells

C
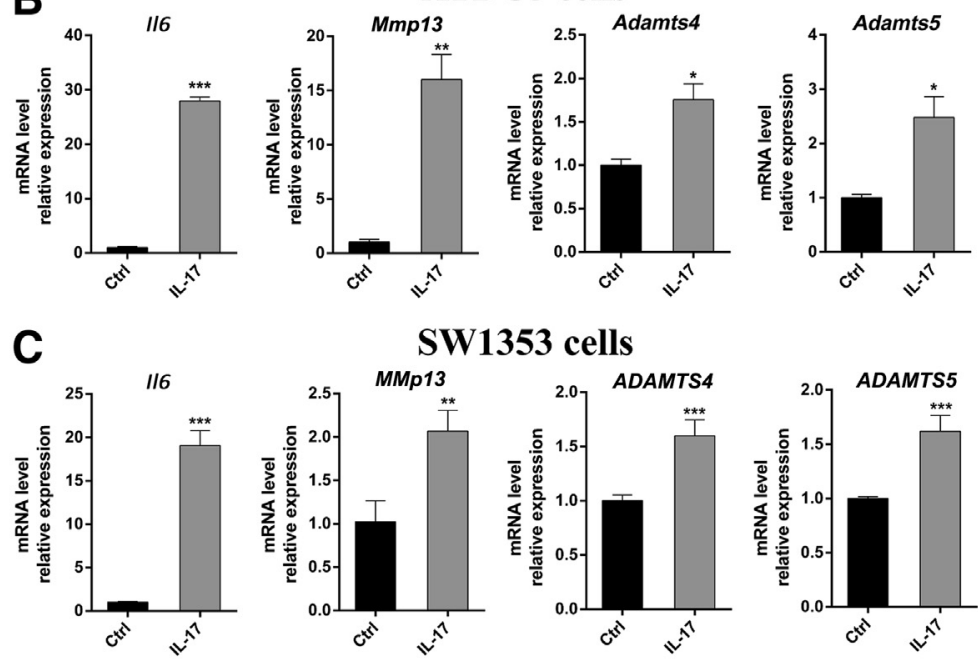

\section{SW1353 cells}
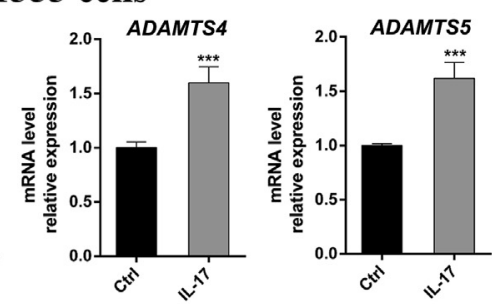

D

E

IL-17 (min): $\quad 0 \quad 1530$

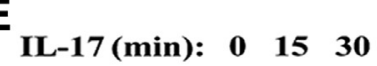

F

IL-17 (min): $015 \quad 30$
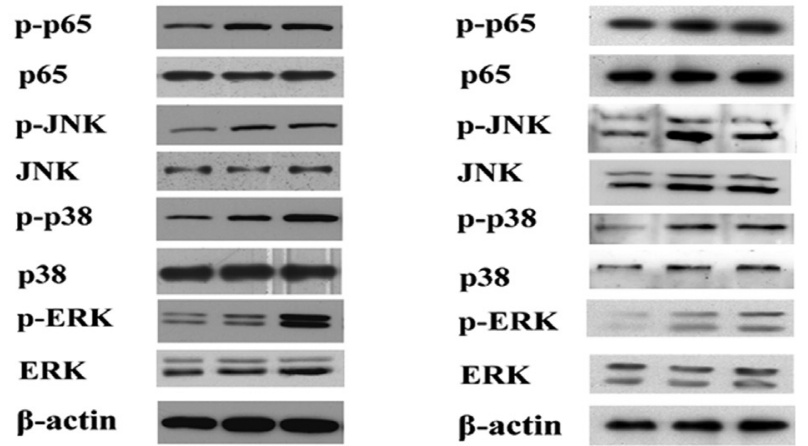

Primary chondrocytes

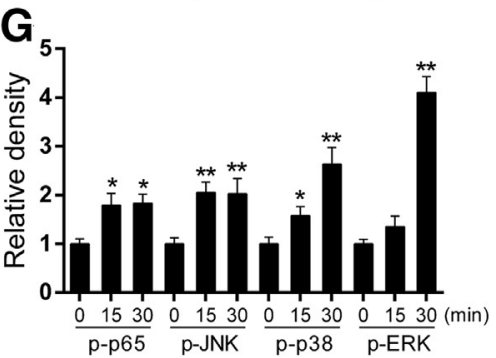

Primary chondrocytes

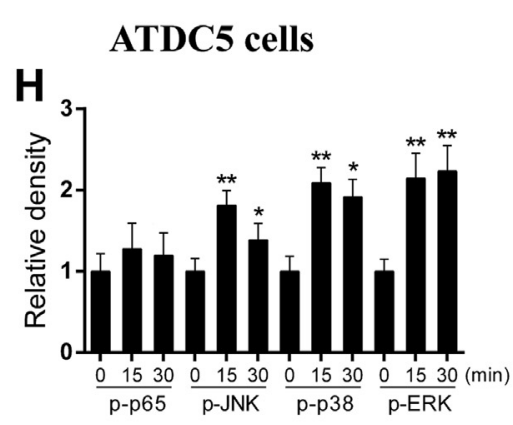

ATDC 5 cells

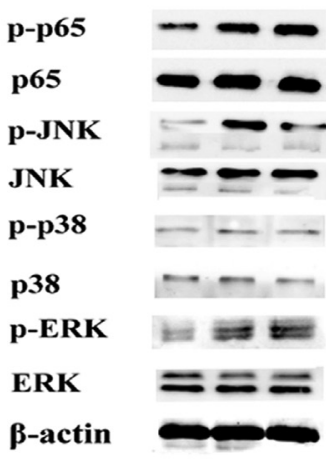

SW1353 cells

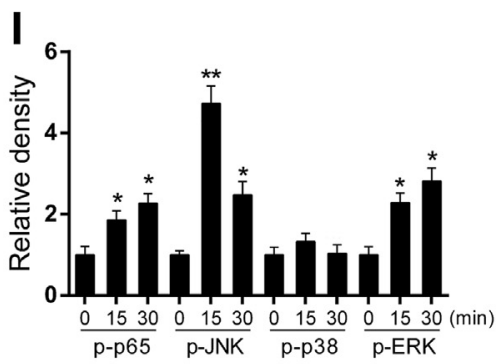

SW1353 cells

Figure 1 Interleukin (IL)-17 activates NF-KB and mitogen activated protein kinase (MAPK) signaling and up-regulates catabolic factors in chondrocytes. A-C: The mRNA levels of catabolic genes in primary rat chondrocytes, the murine ATDC5 cell line, and the human chondrosarcoma SW1353 cell line stimulated with IL-17 (24 hours, 10 $\mathrm{ng} / \mathrm{mL})$. D-F: Immunoblotting of the main molecules involved in the NF- $\mathrm{KB}$ and MAPK signaling pathways in IL-17-stimulated primary chondrocytes, the ATDC5 cell line, and the SW1353 cell line. G-I: Quantifications of the immunoblotting results. Data are expressed as means \pm SEM. $n \geq 3$ independent experiments (A-I). ${ }^{*}<0.05$, ${ }^{*} P<0.01$, and ${ }^{* *} P<0.001$ versus control. Adamts, a disintegrin and metalloproteinase with thrombospondin motifs; ERK, extracellular signal-regulated kinases; JNK, c-Jun N-terminal kinase; min, minutes; MMP, matrix metalloproteinase; $\mathrm{p}$-ERK, phospho-ERK; p-JNK, phospho-JNK. 


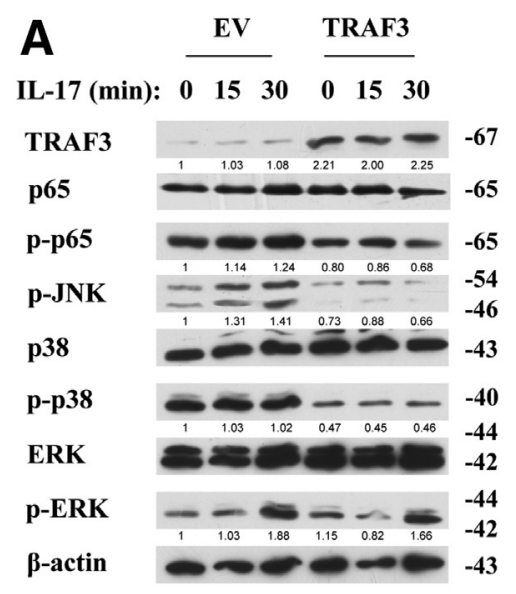

C

Primary chondrocytes

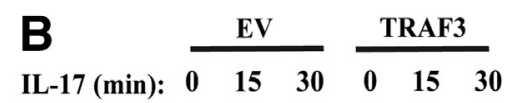

TRAF3

p65

p-p65

p-JNK

p38

p-p38

ERK

p-ERK

$\beta$-actin

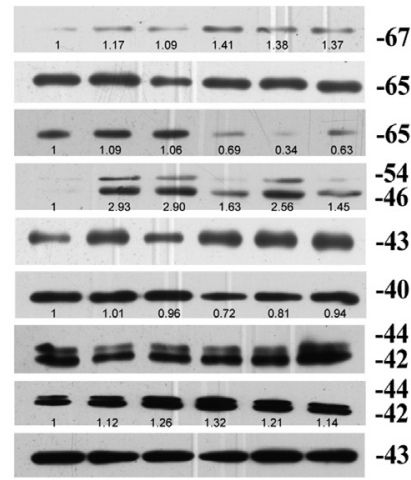

ATDC5 cells

(kDa)

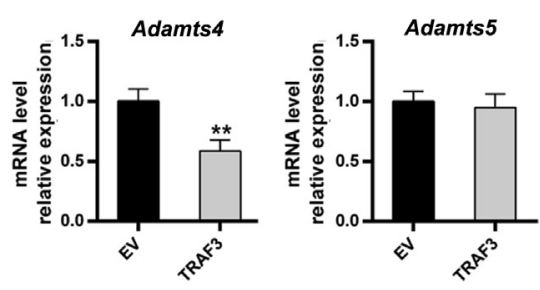

D
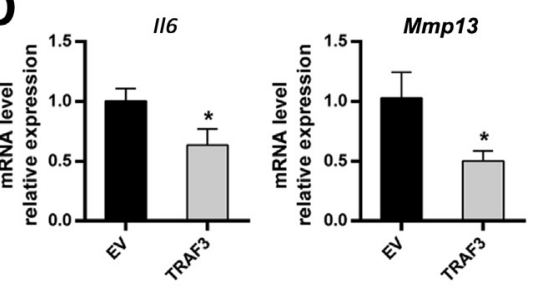

E

(kDa)

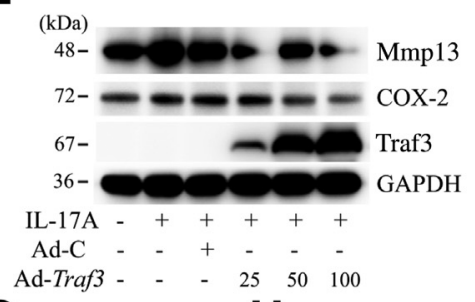

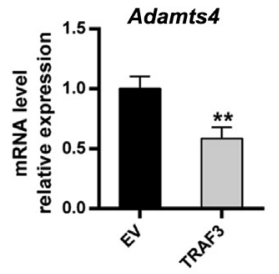

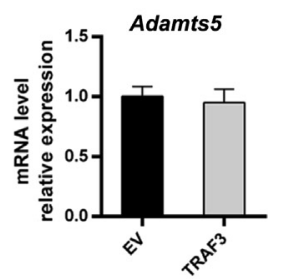

$\mathbf{F}$

(kDa)

ATDC5 cells

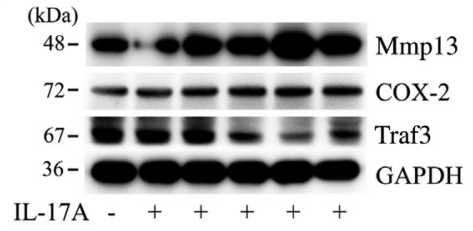

$\begin{array}{ccccccc}\text { IL-17A } & - & + & + & + & + & + \\ \text { Ad-C } & - & - & + & - & - & -\end{array}$

Ad-shTraf3 - $\quad$ - $\quad$ - $25 \quad 50 \quad 100$

I

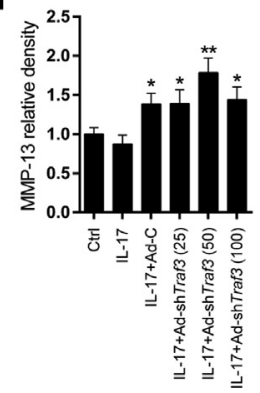

J

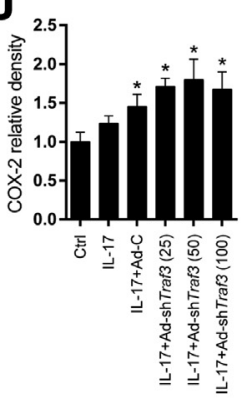

Figure 2 Tumor necrosis factor-receptorassociated factor 3 (Traf3) negatively regulates interleukin (IL)-17-mediated signaling and the induction of matrix-degrading enzymes in chondrocytes. A and B: Primary rat chondrocytes (A) or murine ATDC5 cells (B) transfected with plasmids for empty vector (EV) or M2 (Flag)-tagged TRAF3 were left untreated or treated with IL-17 $(10 \mathrm{ng} / \mathrm{mL})$ for 15 or 30 minutes. Whole-cell lysates were immunoblotted with anti-p65, anti-pp65, anti-p-JNK, anti-p38, anti-p-p38, anti-ERK, anti-p-ERK, anti-TRAF3, or anti- $\beta$-actin. $\mathbf{C}$ and $\mathbf{D}$ : Il6, Mmp13, a disintegrin and metalloproteinase with thrombospondin motifs Adamts4, and Adamts5 mRNA expression induced by IL-17 $(10 \mathrm{ng} / \mathrm{mL})$ in primary chondrocytes (C) or ATDC5 cells infected with adenoviruses containing empty vector $(\mathrm{Ad}-\mathrm{C})$ or adenoviruses containing the entire CDS of TRAF3 (Ad-Traf3) (D). E: Western blot analysis of catabolic factors (Mmp13 and COX-2) in primary chondrocytes infected with Ad-C or Ad-Traf3. F: Immunoblotting of catabolic factors (Mmp13 and COX-2) in primary chondrocytes infected with Ad-C or adenoviruses containing TRAF3 shRNA plasmid (Ad-sh-Traf3). G-J: Quantification of the immunoblotting results. Data are expressed as means \pm SEM. $n \geq 3$ independent experiments (A-J). ${ }^{*} P<0.05,{ }^{* *} P<0.01$ versus control. COX-2, cyclooxygenase-2; Ctrl, control; ERK, extracellular signalregulated kinase; GAPDH, glyceraldehyde-3phosphate dehydrogenase; JNK, c-Jun N-terminal kinase; MMP, matrix metalloproteinase; $p$-ERK, phospho-ERK; p-JNK, phospho-JNK.

cells were infected by viral supernatant suspensions obtained from human embryonic kidney (HEK) 293 FT cells transfected with flag-tagged TRAF3 plasmid together with help vectors. SW1353 cells were transfected by pcDNATRAF3 and empty pcDNA vectors with the use of Lipofectamine 2000 (Invitrogen, Carlsbad, CA).

\section{RNA Interference}

RNA interference was performed using siGENOME SMART pool siRNA (Dharmacon, Lafayette, CO) targeting rat TRAF3 interacting protein 2 (TRAF3IP2 or Act1) (gene ID: 10758). 


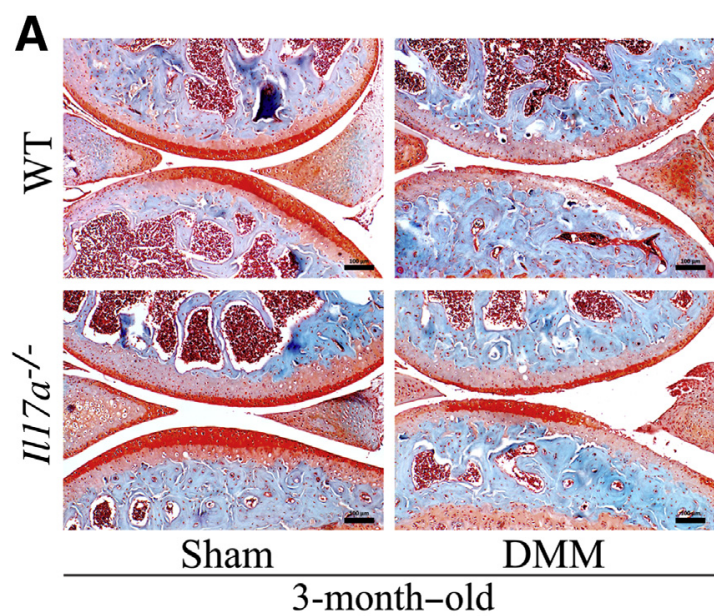

B

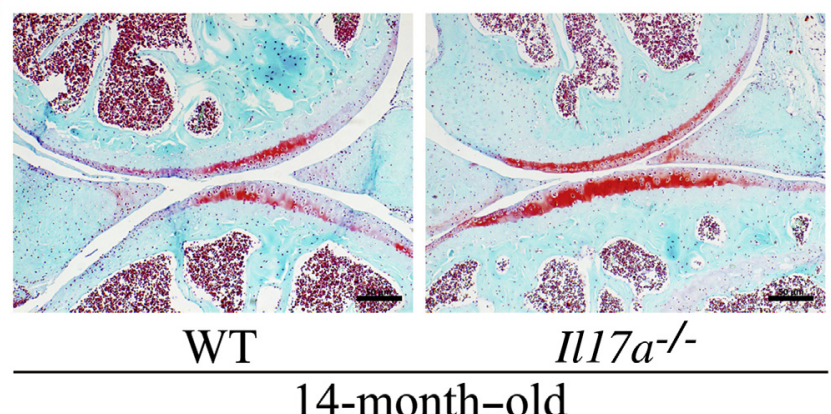

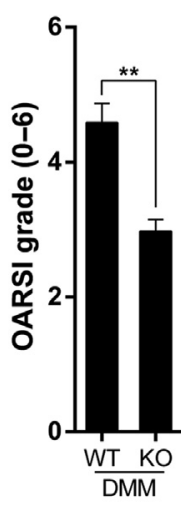

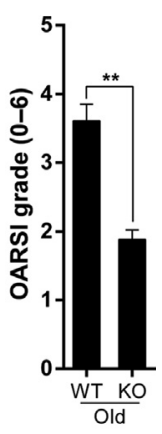

Figure 3 Interleukin (IL)-17 deficiency attenuates cartilage matrix degradation in surgery-induced osteoarthritis (OA) and aging-induced spontaneous OA. A: Cartilage destruction and 0steoarthritis Research Society International (OARSI) grade in sham-operated and destabilization of the medial meniscus (DMM)-operated wild-type (WT) and Il17a-deficient (Il17a $\left.{ }^{-/-}\right)$mice at the age of 3 months. B: Safranin-0 staining and OARSI grade in 14-month-old WT and $\mathrm{Il}_{17 a^{-/}}$mice. Data are expressed as means \pm SEM. $n=6$ (A); $n=8$ (B). ${ }^{* *} P<0.01$ versus control. Scale bars: $100 \mu \mathrm{m}(\mathbf{A}) ; 50 \mu \mathrm{m}$ (B). K0, knockout.

\section{RNA Purification and Quantitative Real-Time PCR}

Total RNA was prepared using TRIzol reagent (Invitrogen) in accordance with the manufacturer's instructions. A first-strand cDNA was synthesized from $1 \mu \mathrm{g}$ of the total RNA by incubating for 1 hour at $42^{\circ} \mathrm{C}$ with Superscript III reverse transcriptase (Invitrogen) after oligo deoxythymine (dT) priming. After the reversetranscription reaction, quantitative real-time PCR was performed with a LightCycler480 system (Roche, Basel, Switzerland) using SYBR1Premix Ex Taq (Takara Bio, Shiga, Japan) in accordance with the manufacturer's instructions. The conditions of real-time PCR were as follows: denaturation at $95^{\circ} \mathrm{C}$ for 10 seconds, 40 cycles at $95^{\circ} \mathrm{C}$ for 10 seconds, and $60^{\circ} \mathrm{C}$ for 30 seconds. The dissociation stage was added to the end of the amplification procedure. No nonspecific amplification was determined by the dissociation curve. All of the amplifications were normalized by $\beta$-actin. Data were analyzed using the comparison $\mathrm{Ct}\left(2^{-\triangle \Delta \mathrm{Ct}}\right)$ method and expressed as fold change compared with the respective control.

\section{Western Blot}

Cells were lysed on ice for 30 minutes in the lysis buffer containing $50 \mathrm{mmol} / \mathrm{L}$ Tris- $\mathrm{HCl}, \mathrm{pH} 7.4,150 \mathrm{mmol} / \mathrm{L} \mathrm{NaCl}$, $1 \%$ Nonidet P-40, and $0.1 \%$ SDS supplemented with protease inhibitors $(10 \mathrm{mg} / \mathrm{mL}$ leupeptin, $10 \mathrm{mg} / \mathrm{mL}$ pepstatin
$\mathrm{A}$, and $10 \mathrm{mg} / \mathrm{mL}$ aprotinin). For Western blot analysis, a 50 $\mu \mathrm{g}$ protein sample was resolved on 10\% SDS-PAGE and electrotransferred onto nitrocellulose membranes (Whatman, Piscataway, NJ). The primary antibodies used were antiTRAF3 (1:1000; Abcam), MMP-13 (1:200, sc-30073; Santa Cruz Biotechnology, Santa Cruz, CA), cyclooxygenase (COX)-2 (1:500, ab15191; Abcam), glyceraldehyde-3phosphate dehydrogenase (1:5000, G9545; Sigma), anti-phospho-p65 (1:1000), anti-p65 (1:1000), anti-phosphop38 MAPK (1:1000), anti-p38 MAPK (1:1000), anti-phospho-p44/42 MAPK (1:2000), anti-p44/42 MAPK (1:1000), anti-phospho-c-Jun N-terminal kinase (JNK) (1:1000), and anti-JNK (1:1000), which all were purchased from Cell Signaling Technology. $\beta$-actin was used as a loading control. Horseradish-peroxidase-conjugated secondary antibodies (Cell Signaling Technology) were used at a 1:1000 dilution. The antigen-antibody complexes were made visible using an enhanced chemiluminescence detection system in accordance with the manufacturer's recommendations. Immunoreactive bands were analyzed quantitatively in triplicate by normalizing the band intensities into $\beta$-actin on scanned films with the use of ImageJ.

\section{Statistical Analysis}

Statistical analyses were performed using GraphPad Prism software version 6 (GraphPad, Durham, NC). All data are 
A

WT

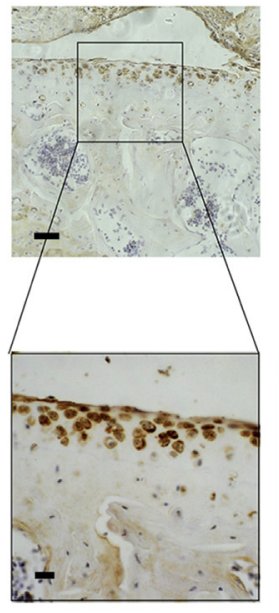

C
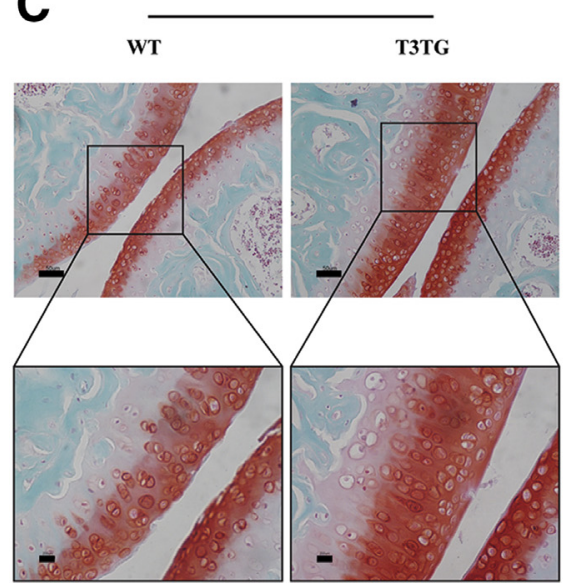

PBS

WT T3TG

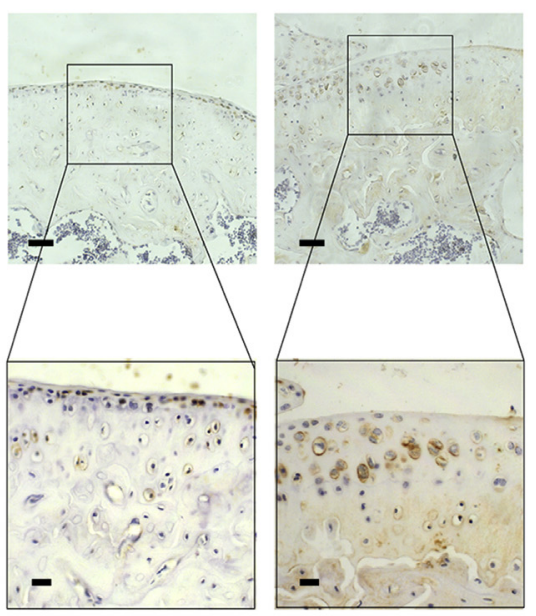

PBS

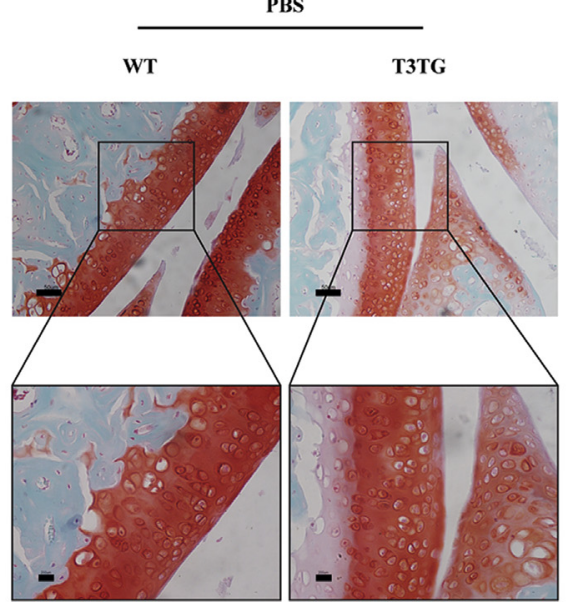

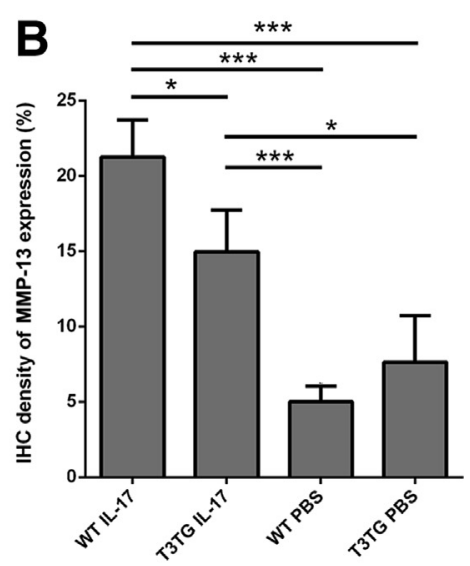

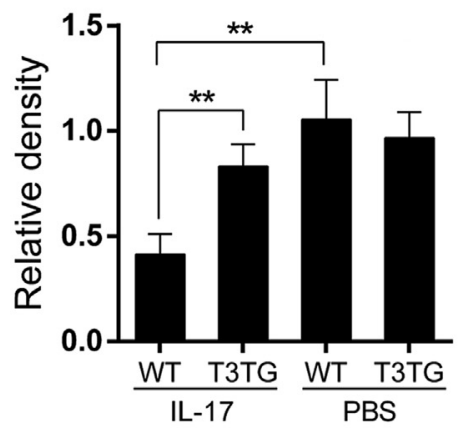

Figure 4 Tumor necrosis factor-receptor-associated factor 3 (TRAF3) transgenic mice (T3TG) show less matrix metalloproteinase 13 (MMP-13) production and cartilage extracellular matrix loss induced by interleukin (IL)-17. A and B: Representative images and quantification of immunohistochemistry of MMP-13 in T3TG and littermate control mice after intra-articular injection of IL-17 or phosphate-buffered saline (PBS). Boxed region shows MMP-13-positive chondrocytes. C: Safranin-0 staining of the articular cartilage of T3TG and littermate control after intra-articular injection of IL-17 or PBS. Boxed region shows magnified articular cartilage. Data are expressed as means \pm SEM. $n=6$. ${ }^{*} P<0.05,{ }^{*} P<0.01$, and ${ }^{*} * P<0.001$. Scale bars: $50 \mu \mathrm{m}(\mathbf{A}$ and $\mathbf{C}) ; 20 \mu \mathrm{m}$ (A and C, higher magnification). IHC, immunohistochemistry; WT, wild-type.

shown as means \pm SD. Statistical differences between two groups were determined by a two-tailed $t$-test. $P$ values less than 0.05 were considered statistically significant. All experiments were performed on at least 3 individual samples.

\section{Results}

\section{IL-17 Induces the Production of Inflammatory} Cytokines, MMPs, and Aggrecanase in Chondrocytes

To investigate the role of IL-17 in chondrocytes catabolism, chondrocytes were treated with recombinant IL-17 (10 ng/ $\mathrm{mL}, 24$ hours). IL-17 treatment resulted in significant up-regulation of IL6, MMP13, a disintegrin and metalloproteinase with thrombospondin motifs-4 (ADAMTS4) and ADAMTS5 at the mRNA level, which play crucial roles in OA cartilage destruction in rat primary chondrocytes (Figure 1A), ATDC5 cells (Figure 1B), as well as SW1353 cells (Figure 1C). Moreover, treatment of IL-17 promptly triggered the phosphorylation of p65, p38, extracellular signal-regulated kinase (ERK), and JNK in rat primary chondrocytes (Figure 1, D and G) and ATDC5 cells (Figure 1, E and H). However, IL-17 only activated the phosphorylation of p65, ERK, and JNK, but not p38 in SW1353 cells (Figure 1, F and I). Altogether, these data suggest that IL-17 up-regulates cartilage catabolic factors through activation of NF- $\mathrm{KB}$ and MAPK signaling. 
A

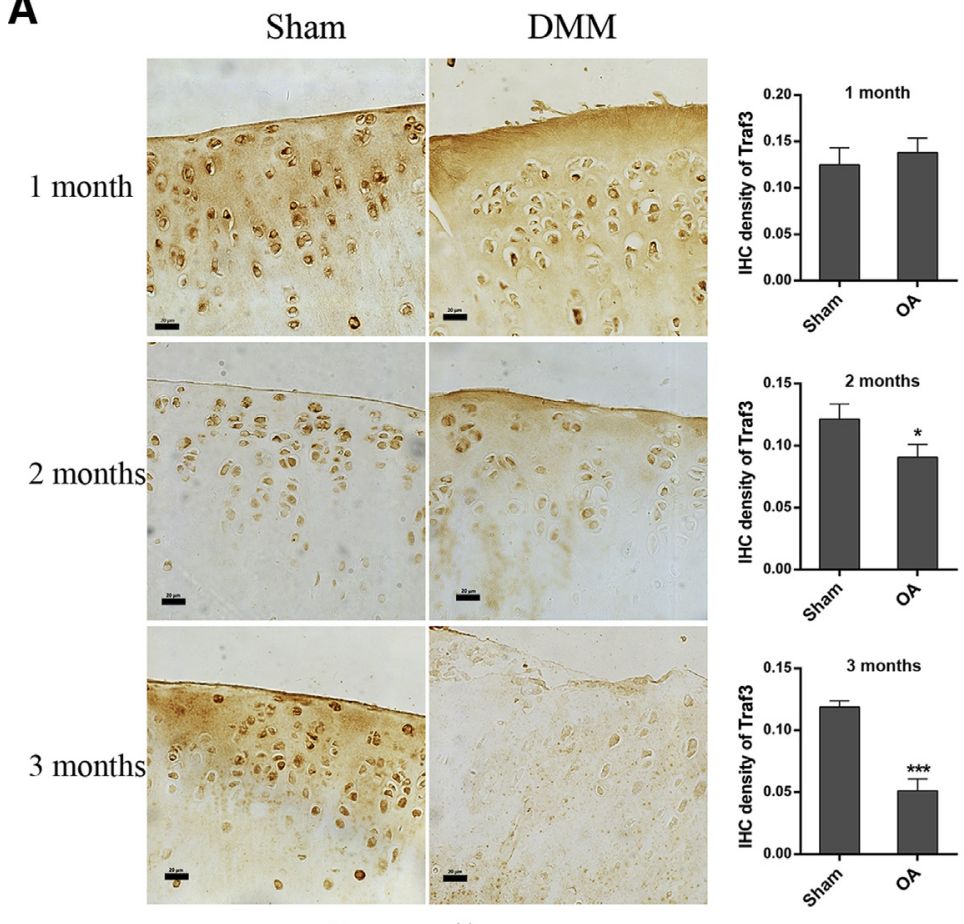

Rat cartilage

B

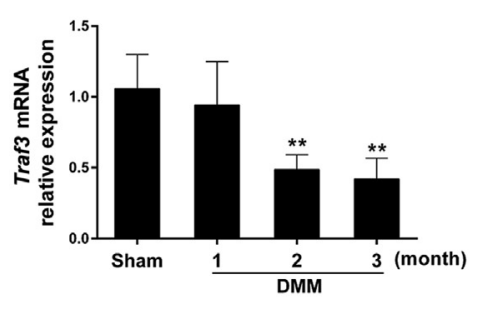

C

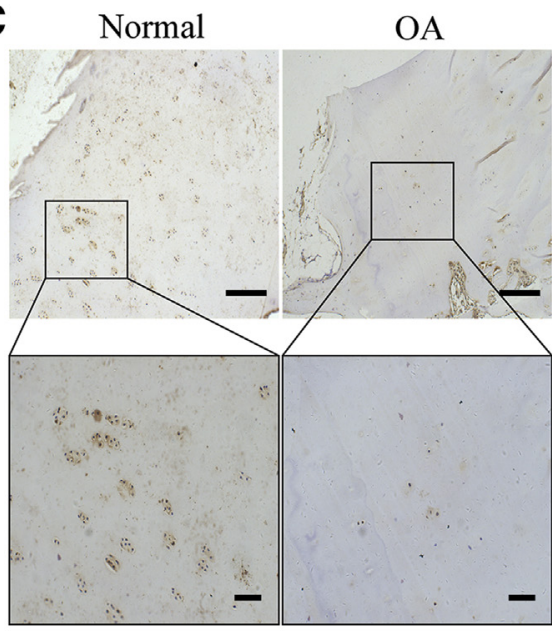

Human cartilage
Figure 5 Increased tumor necrosis factorreceptor-associated factor 3 (Traf3) expression during experimental osteoarthritis (OA) pathogenesis. A: Immunohistochemistry of Traf3 expression in rat cartilage obtained from destabilization of the medial meniscus (DMM)-induced experimental $\mathrm{OA}$ or sham groups at 1 month, 2 months, and 3 months after surgery. B: Fold change of Traf3 mRNA expression level in cartilage obtained from the rat described in panel A. C: Immunohistochemistry of TRAF3 expression in cartilage obtained from $0 \mathrm{~A}$ patients. Boxed region shows TRAF3 positive chondrocytes. Data are expressed as means \pm SEM. $n=8$ (A, 1 month); $n=6$ (A, 2 months); $n=9$ (A, 3 months); $n=3$ (C). ${ }^{*} P<0.05,{ }^{*} P<<0.01$, and ${ }^{* *} P<0.001$. Scale bars: $20 \mu \mathrm{m}$ (A, C, higher magnification); 50 $\mu \mathrm{m}(\mathrm{C})$. IHC, immunohistochemistry.

\section{TRAF3 Tightly Controls IL-17-Induced Expression of Catabolic Genes}

To determine the role of TRAF3 in IL-17-mediated signaling, an adenovirus containing the entire coding sequence (CDS) sequence of TRAF3 (Ad-Traf3) was generated. Indeed, Ad-Traf3 infection effectively increased the TRAF3 expression level in primary chondrocytes (Supplemental Figure S1, A and B). Overexpression of TRAF3 notably suppressed IL-17-induced p65 phosphorylation, p38 phosphorylation, JNK phosphorylation, and ERK phosphorylation, indicating that TRAF3 has general inhibitory effects on IL-17-mediated pathways (Figure 2A). Moreover, TRAF3 or empty vector plasmids were also transfected into SW1353 cells and IL17-mediated immediate signaling was checked. Consistently, IL-17-induced p65 phosphorylation, p38 phosphorylation, and JNK phosphorylation, but not ERK phosphorylation, were inhibited by TRAF3 overexpression (Figure 2B), indicating that TRAF3 has general inhibitory effects on IL-17-mediated pathways in chondrocytes. This study then investigated the role of TRAF3 in IL17-induced expression of cartilage catabolic factors in chondrocytes. Notably, TRAF3 overexpression suppressed 
A

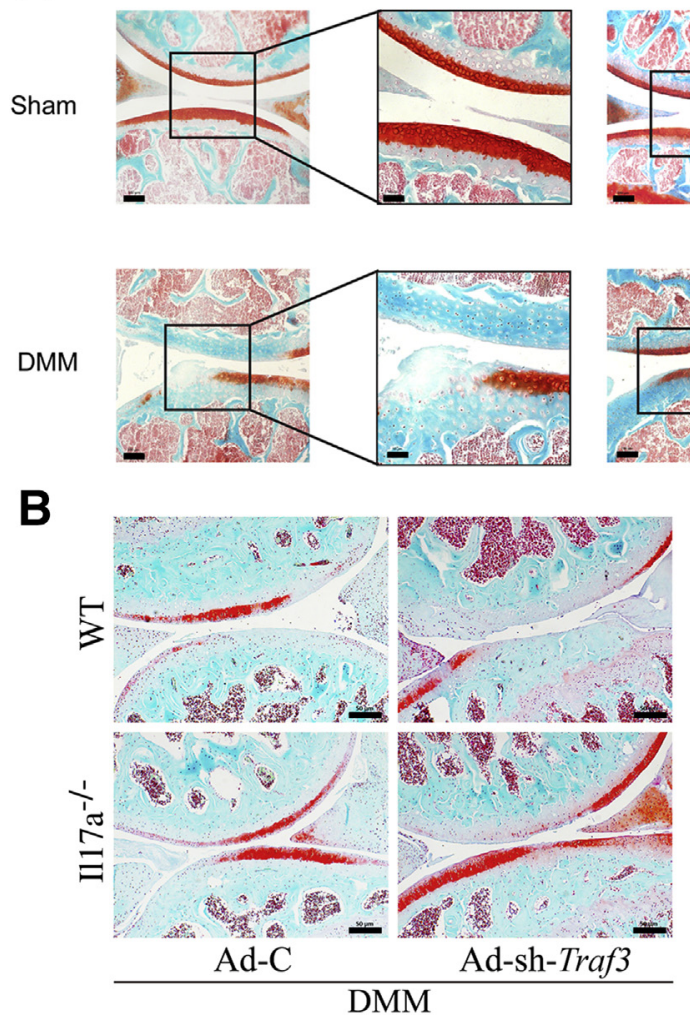

T3TG
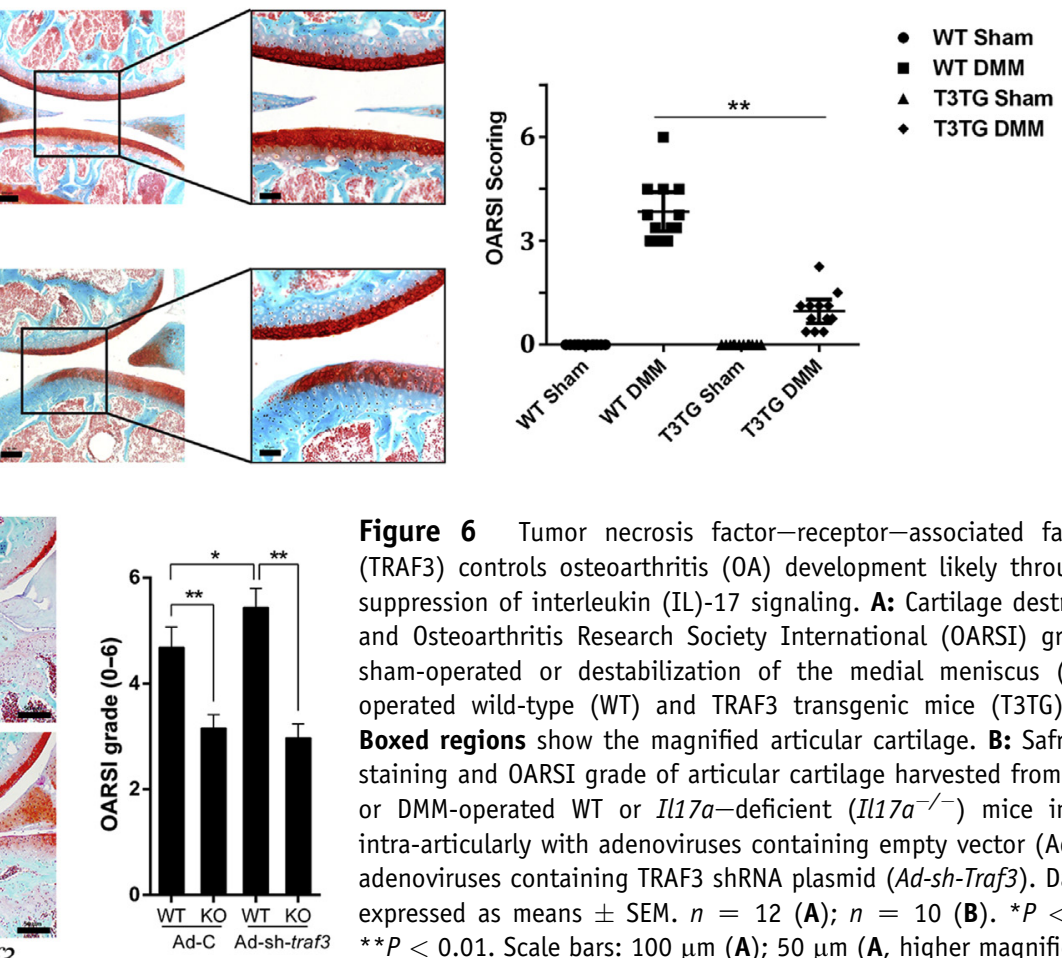

Figure 6 Tumor necrosis factor-receptor-associated factor 3 (TRAF3) controls osteoarthritis (OA) development likely through its suppression of interleukin (IL)-17 signaling. A: Cartilage destruction and 0steoarthritis Research Society International (OARSI) grade in sham-operated or destabilization of the medial meniscus (DMM)operated wild-type (WT) and TRAF3 transgenic mice (T3TG) mice. Boxed regions show the magnified articular cartilage. B: Safranin-0 staining and OARSI grade of articular cartilage harvested from sham-

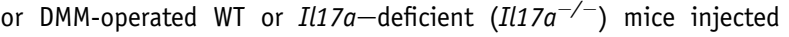
intra-articularly with adenoviruses containing empty vector (Ad-C) or adenoviruses containing TRAF3 shRNA plasmid (Ad-sh-Traf3). Data are expressed as means \pm SEM. $n=12(\mathbf{A}) ; n=10$ (B). ${ }^{*} P<0.05$, $* * P<0.01$. Scale bars: $100 \mu \mathrm{m}(\mathbf{A}) ; 50 \mu \mathrm{m}(\mathbf{A}$, higher magnification, and B). K0, knockout.

IL-17-induced up-regulation of Il6, Mmp13, and Adamts4, but not Adamts5, at the mRNA level in both primary chondrocytes and ATDC5 cells (Figure 2, C and D). TRAF3 overexpression also significantly reduced the protein level of Mmp13 and COX-2 under the stimulation of IL-17 (Figure 2, E, G, and H). To further confirm the role of TRAF3 in IL-17-mediated induction of downstream genes, Traf3-specific siRNA were generated. These data showed that Traf3 expression was reduced efficiently at both the mRNA and protein levels in primary chondrocytes (Supplemental Figure S1, C and D). As expected, TRAF3 loss of function enhanced IL-17-induced up-regulation of MMP-13 and COX-2 at the protein level (Figure 2, F, I, and $\mathrm{J})$. Considering that TRAF3 interfered with the binding of IL-17 receptor with Act1, and consequently blocked the recruitment of TRAF6, finally resulting in a suppression of IL-17-mediated signaling and downstream gene transcription, ${ }^{22}$ three $A c t 1$ specific siRNAs were therefore generated to knock down Act1 in chondrocytes. siRNA3 was used for the following investigations because of its highest knockdown efficiency (Supplemental Figure S1, E and F). Importantly, knockdown of Act1 dramatically reduced the up-regulation of the MMP-13 protein level induced by IL17 (Supplemental Figure S1G). Collectively, these data solidly show that TRAF3 critically controls IL-17-induced downstream catabolic gene expression in chondrocytes.

\section{IL-17 Deficiency Protects Cartilage from Degradation in Experimental and Spontaneous OA}

Next, this study examined further whether blocking IL-17 signaling can protect cartilage from degradation in vivo. Interestingly, cartilage destruction triggered by DMM surgery was alleviated significantly in IL-17-deficient mice compared with littermate controls (Figure 3A). Furthermore, IL-17 depletion also notably mitigated cartilage degradation caused by age-related spontaneous OA (Figure 3B). Collectively, these results suggest that IL-17 plays a critical role in cartilage degradation during $\mathrm{OA}$.

\section{TRAF3 Transgenic Mice Show Less Matrix Loss Induced} by IL-17

To investigate the role of TRAF3 in IL-17-induced cartilage degradation in vivo, T3TG mice and littermate controls were used to generate an IL-17 intra-articular injection model. MMP-13 is a critical catabolic factor during cartilage extracellular matrix loss and cartilage degradation. Indeed, an obvious increase of IL-17 injection-induced Mmp13 production was detected in the superficial cartilage zone of WT mice, which was inhibited significantly in the cartilage of T3TG mice (Figure 4, A and B). Consistently, cartilage extracellular matrix loss, which was reflected by 
safranin-O staining, caused by IL-17 injection, was attenuated in T3TG mice compared with littermate controls (Figure 4C). Taken together, these results proved that TRAF3 attenuates IL-17-induced MMP-13 production, and thus inhibits cartilage degradation in vivo.

\section{TRAF3 Expression Is Reduced in OA Cartilage}

To determine whether the TRAF3 expression level in articular cartilage is altered during $\mathrm{OA}$, an experimental $\mathrm{OA}$ rat model was generated to investigate the expression of Traf 3 in $\mathrm{OA}$ and normal cartilage. The immunohistochemistry results showed that the Traf3 expression level in experimental OA cartilage was slightly reduced 1 month after surgery and decreased significantly after 2 and 3 months compared with the sham group (Figure 5A). Meanwhile, the mRNA level of Traf3 in cartilage was analyzed at different stages of the rat experimental OA model and showed that TRAF3 was reduced significantly during the OA process (Figure 5B). A similar observation that the TRAF3 expression level in OA cartilage was lower than that of normal cartilage also was found in human OA patients (Figure 5C). In summary, these observations indicate that TRAF3 is down-regulated in cartilage during OA pathogenesis.

\section{TRAF3 Plays a Protective Role in Cartilage Degradation Caused by $0 \mathrm{~A}$}

Considering that TRAF3 has an inhibitory role in IL-17-mediated signaling and induction of matrixdegrading enzymes in vitro, this study further investigated whether TRAF3 affects experimental OA pathogenesis in vivo. To this end, an experimental $\mathrm{OA}$ model was generated by DMM surgery in T3TG mice and littermate controls. One month after the surgery, TRAF3 deficiency effectively alleviated cartilage destruction triggered by DMM surgery, which was shown by histology and Osteoarthritis Research Society International scoring (Figure 6A). To confirm further if TRAF3-mediated suppression of IL-17 signaling is responsible for its protective effect on cartilage degradation, Ad-sh-Traf 3 were injected intra-articularly into the knee joint of mice with DMM-induced OA. The results showed that loss of TRAF3 worsened cartilage destruction caused by surgery-induced OA, however, Ad-sh-Traf3 had no effect on cartilage degradation caused by DMM surgery in $1 l 17 a^{-1-}$ mice (Figure 6B). In summary, our results suggest that TRAF3-mediated suppression of IL-17 signaling is a potential effective therapeutic target for cartilage matrix degradation in OA.

\section{Discussion}

IL-17 has been found to activate various signaling pathways for robust induction of proinflammatory cytokines and chemokines. ${ }^{4,13}$ Moreover, IL-17 also can act synergistically with IL-1 $\beta$ or TNF- $\alpha$ for further induction of proinflammatory cytokines, nitric oxide, and MMPs, ${ }^{14}$ as well as reduction of the proteoglycan level. ${ }^{28}$ Indeed, IL-17, acting as an upstream factor, significantly increased the expression of IL-6, MMP-13, ADAMTS-4, and ADAMTS5 in primary chondrocytes, the ATDC5 cell line, and the SW1353 cell line. However, the up-regulation of these catabolic factors induced by IL-17 is relatively modest compared with IL-1 $\beta$ or TNF- $\alpha$, suggesting that IL-17 might not be a direct regulator of these catabolic factors, rather, IL-17 might up-regulate IL-1 $\beta$ or TNF- $\alpha$ through the activation of NF- $\kappa \mathrm{B}$ and MAPK pathways to turn on the expression of catabolic factors. Similarly, in vivo local administration of IL-17 induced MMP-13 production in articular cartilage, accompanied by cartilage matrix loss. However, only mild matrix loss was observed in the IL-17 injection model, which might be attributed to the short IL17 exposure time course (3 days). Most importantly, IL-17 global loss-of-function mice showed attenuated cartilage degradation during surgery-induced OA and aging-related spontaneous OA, suggesting that IL-17 might be an important mediator of cartilage degradation during OA. Therefore, blocking IL-17-mediated downstream signaling in chondrocytes is a potent strategy to protect cartilage from degradation.

TRAF3 was identified as an important negative regulator of the noncanonical NF- $\kappa \mathrm{B}$ pathway in multiple cell types. ${ }^{19,29}$ A previous study showed that IL-17 stimulation induces the recruitment of TRAF3 to IL-17 receptor, which interferes with the formation of the $\mathrm{IL}-17$ receptor-Act1-TRAF6 activation complex. ${ }^{22}$ A previous study showed that TRAF3 suppressed IL-17-mediated signaling in primary synoviocytes. ${ }^{22}$ Consistently, TRAF3 overexpression notably suppressed IL-17-mediated phosphorylation of p65, JNK, ERK, and p38, and consequent production of IL-6, MMP-13, Adamts-4, and COX-2, but not Adamts-5 in primary chondrocytes in the current study. However, TRAF3 overexpression only suppressed p65 and JNK phosphorylation in the ATDC5 cell line, indicating the heterogeneity of TRAF3 function in different cell types. To confirm the protective role of TRAF3 in IL-17-mediated cartilage degradation in vivo, an IL-17 intra-articular injection model was established. Importantly, TRAF3 gainof-function effectively reduced IL-17-induced MMP-13 production and matrix loss in mice. Interestingly, TRAF3 overexpression does not affect the Adamts-5 expression level in both primary chondrocytes and the ATDC5 cell line. Adamts-5 was thought to be the main aggrecanase in mice, ${ }^{30}$ therefore, TRAF3 gain of function might not be able to attenuate aggrecan degradation in mice.

The cartilage matrix loss and cartilage degradation of T3TG mice were attenuated compared with the WT mice. According to the Osteoarthritis Research Society International scoring, in T3TG mice, modest cartilage extracellular matrix loss still was observed after the surgery (grades 1 to 2). However, obvious cartilage degradation was not observed in T3TG mice, whereas the WT cartilage underwent notable degradation (grades 2 to 5). 
In addition, the involvement of surface area in T3TG mice (stages 1 to 2) was less than that in the WT mice (stages 3 to 4). On the other hand, TRAF3 loss of function enhanced cartilage destruction caused by DMM surgery. Interestingly, the TRAF3 expression level decreased significantly at the late stage (from 2 months after DMM surgery) of OA pathogenesis but not the early stage in rat OA cartilage. By 3 months after DMM surgery, TRAF3 barely was detected in articular cartilage (Figure 5A). The mRNA expression further confirmed the significant change of TRAF3 during OA, suggesting that TRAF3 is down-regulated transcriptionally and leads to enhanced IL-17 signaling in cartilage during OA pathogenesis. These findings indicate that TRAF3 is a critical negative regulator of cartilage degradation during OA. More importantly, genetic deletion of IL-17 blocked TRAF3 knockdown-mediated promotion of cartilage destruction. These data suggest that the protective effect of TRAF3 on cartilage is mediated by its suppression of IL-17-mediated signaling.

In summary, we show that TRAF3, as a negative regulator of IL-17-mediated signaling, plays an important role in protecting articular cartilage from degradation during OA. The protective effect of TRAF3 on cartilage is partially owing to the suppression of IL-17-induced signaling and consequent production of cartilage catabolic factors. Thus, TRAF3 is a potential therapeutic target for the development of a treatment for cartilage degradation caused by OA.

\section{Acknowledgment}

We thank Prof. Youcun Qian (Chinese Academy of Sciences) for kindly providing the T3TG and $1117 a^{-1-}$ mice.

\section{Author Contributions}

G.H. and N.Z. conceived and designed the study, wrote the manuscript, and collected, analyzed, and interpreted the data; J.L. collected and analyzed the data; J.W. performed animal surgery and collected data; W.W. collected human cartilage samples; J.L. wrote the manuscript; W.T. and X.Z. collected and analyzed the data; L.D. analyzed the data; and X.Z. conceived and designed the study, wrote the manuscript, and analyzed and interpreted the data.

\section{Supplemental Data}

Supplemental material for this article can be found at http://doi.org/10.1016/j.ajpath.2020.04.016.

\section{References}

1. Loeser RF, Goldring SR, Scanzello CR, Goldring MB: Osteoarthritis: a disease of the joint as an organ. Arthritis Rheum 2012, 64:1697-1707

2. Tong W, Geng Y, Huang Y, Shi Y, Xiang S, Zhang N, Qin L, Shi Q, Chen Q, Dai K, Zhang X: In vivo identification and induction of articular cartilage stem cells by inhibiting NF-kappaB signaling in osteoarthritis. Stem Cells 2015, 33:3125-3137
3. Benito MJ, Veale DJ, FitzGerald O, van den Berg WB, Bresnihan B: Synovial tissue inflammation in early and late osteoarthritis. Ann Rheum Dis 2005, 64:1263-1267

4. Kapoor M, Martel-Pelletier J, Lajeunesse D, Pelletier JP, Fahmi H: Role of proinflammatory cytokines in the pathophysiology of osteoarthritis. Nat Rev Rheumatol 2011, 7:33-42

5. Pelletier JP, Martel-Pelletier J, Abramson SB: Osteoarthritis, an inflammatory disease: potential implication for the selection of new therapeutic targets. Arthritis Rheum 2001, 44:1237-1247

6. Komiyama Y, Nakae S, Matsuki T, Nambu A, Ishigame H, Kakuta S, Sudo K, Iwakura Y: IL-17 plays an important role in the development of experimental autoimmune encephalomyelitis. J Immunol 2006, 177 : $566-573$

7. Nakae S, Nambu A, Sudo K, Iwakura Y: Suppression of immune induction of collagen-induced arthritis in IL-17-deficient mice. J Immunol 2003, 171:6173-6177

8. Sato K, Suematsu A, Okamoto K, Yamaguchi A, Morishita Y, Kadono Y, Tanaka S, Kodama T, Akira S, Iwakura Y: Th17 functions as an osteoclastogenic helper $\mathrm{T}$ cell subset that links $\mathrm{T}$ cell activation and bone destruction. J Exp Med 2006, 203:2673-2682

9. Ye P, Rodriguez FH, Kanaly S, Stocking KL, Schurr J, Schwarzenberger P, Oliver P, Huang W, Zhang P, Zhang J, Shellito JE, Bagby GJ, Nelson S, Charrier K, Peschon JJ, Kolls JK: Requirement of interleukin 17 receptor signaling for lung CXC chemokine and granulocyte colony-stimulating factor expression, neutrophil recruitment, and host defense. J Exp Med 2001, 194:519-527

10. Ruddy MJ, Wong GC, Liu XK, Yamamoto H, Kasayama S, Kirkwood KL, Gaffen SL: Functional cooperation between interleukin-17 and tumor necrosis factor-alpha is mediated by CCAAT/enhancer-binding protein family members. J Biol Chem 2004, 279:2559-2567

11. Shen F, Ruddy MJ, Plamondon P, Gaffen SL: Cytokines link osteoblasts and inflammation: microarray analysis of interleukin-17- and TNFalpha-induced genes in bone cells. J Leukoc Biol 2005, 77:388-399

12. Hwang SY, Kim JY, Kim KW, Park MK, Moon Y, Kim WU, Kim HY: IL-17 induces production of IL-6 and IL-8 in rheumatoid arthritis synovial fibroblasts via NF-kappaB- and PI3-kinase/Aktdependent pathways. Arthritis Res Ther 2004, 6:R120-R128

13. Shalom-Barak T, Quach J, Lotz M: Interleukin-17-induced gene expression in articular chondrocytes is associated with activation of mitogen-activated protein kinases and NF-kappaB. J Biol Chem 1998, 273:27467-27473

14. Martel-Pelletier J, Mineau F, Jovanovic D, Di Battista JA, Pelletier JP: Mitogen-activated protein kinase and nuclear factor kappaB together regulate interleukin-17-induced nitric oxide production in human osteoarthritic chondrocytes: possible role of transactivating factor mitogen-activated protein kinase-activated protein kinase (MAPKAPK). Arthritis Rheum 1999, 42:2399-2409

15. Attur MG, Patel RN, Abramson SB, Amin AR: Interleukin-17 upregulation of nitric oxide production in human osteoarthritis cartilage. Arthritis Rheum 1997, 40:1050-1053

16. Honorati MC, Bovara M, Cattini L, Piacentini A, Facchini A: Contribution of interleukin 17 to human cartilage degradation and synovial inflammation in osteoarthritis. Osteoarthritis Cartilage 2002, 10:799-807

17. Alzabin S, Abraham SM, Taher TE, Palfreeman A, Hull D, McNamee K, Jawad A, Pathan E, Kinderlerer A, Taylor PC, Williams R, Mageed R: Incomplete response of inflammatory arthritis to TNFalpha blockade is associated with the Th17 pathway. Ann Rheum Dis 2012, 71:1741-1748

18. Schneider K, Benedict CA, Ware CF: A TRAFfic cop for host defense. Nat Immunol 2006, 7:15-16

19. Yao Z, Xing L, Boyce BF: NF-kappaB p100 limits TNF-induced bone resorption in mice by a TRAF3-dependent mechanism. J Clin Invest 2009, 119:3024-3034

20. Oganesyan G, Saha SK, Guo B, He JQ, Shahangian A, Zarnegar B, Perry A, Cheng G: Critical role of TRAF3 in the Toll-like receptor-dependent and -independent antiviral response. Nature 2006, 439:208-211 
21. Hacker H, Redecke V, Blagoev B, Kratchmarova I, Hsu LC, Wang GG, Kamps MP, Raz E, Wagner H, Hacker G, Mann M, Karin M: Specificity in Toll-like receptor signalling through distinct effector functions of TRAF3 and TRAF6. Nature 2006, 439:204-207

22. Zhu S, Pan W, Shi PQ, Gao HC, Zhao F, Song XY, Liu Y, Zhao LH, Li XX, Shi YF, Qian YC: Modulation of experimental autoimmune encephalomyelitis through TRAF3-mediated suppression of interleukin 17 receptor signaling. J Exp Med 2010, 207:2647-2662

23. Dudler J, Renggli-Zulliger N, Busso N, Lotz M, So A: Effect of interleukin 17 on proteoglycan degradation in murine knee joints. Ann Rheum Dis 2000, 59:529-532

24. Glasson SS, Blanchet TJ, Morris EA: The surgical destabilization of the medial meniscus (DMM) model of osteoarthritis in the 129/SvEv mouse. Osteoarthritis Cartilage 2007, 15:1061-1069

25. Li J, Huang JG, Dai LM, Yu DG, Chen Q, Zhang XL, Dai KR: miR146a, an IL-1 beta responsive miRNA, induces vascular endothelial growth factor and chondrocyte apoptosis by targeting Smad4. Arthritis Res Ther 2012, 14:R75
26. Pritzker KP, Gay S, Jimenez SA, Ostergaard K, Pelletier JP, Revell PA, Salter D, van den Berg WB: Osteoarthritis cartilage histopathology: grading and staging. Osteoarthritis Cartilage 2006, 14:13-29

27. Glasson SS, Chambers MG, Van Den Berg WB, Little CB: The OARSI histopathology initiative - recommendations for histological assessments of osteoarthritis in the mouse. Osteoarthritis Cartilage 2010, 18 Suppl 3:S17-S23

28. Lubberts E, Joosten LA, van de Loo FA, van den Gersselaar LA, van den Berg WB: Reduction of interleukin-17-induced inhibition of chondrocyte proteoglycan synthesis in intact murine articular cartilage by interleukin-4. Arthritis Rheum 2000, 43:1300-1306

29. de Jong SJ, Albrecht J-C, Giehler F, Kieser A, Sticht H, Biesinger B: Noncanonical NF-\{kappa\} B activation by the oncoprotein tio occurs through a nonconserved TRAF3-binding motif. Sci Signal 2013, 6:ra27

30. Glasson SS, Askew R, Sheppard B, Carito B, Blanchet T, Ma HL, Flannery CR, Peluso D, Kanki K, Yang Z, Majumdar MK, Morris EA: Deletion of active ADAMTS5 prevents cartilage degradation in a murine model of osteoarthritis. Nature 2005, 434:644-648 